\title{
Comprehensive RNA expression profile of therapeutic adipose-derived mesenchymal stem cells co-cultured with degenerative nucleus pulposus cells
}

\author{
ZHIHUA HAN $^{1,2}$, QIUGEN WANG $^{1}$, XIAOMING WU $^{1}$, JIANDONG WANG ${ }^{1}$, \\ LIANG GAO ${ }^{2,3}$, RUIPENG GUO ${ }^{2,4}$ and JIANHONG WU ${ }^{1}$
}

\begin{abstract}
${ }^{1}$ Trauma Centre, Department of Trauma and Orthopedics, Shanghai General Hospital, Shanghai Jiao Tong University, Shanghai 201620, P.R. China; ${ }^{2}$ Sino Euro Orthopaedics Network; ${ }^{3}$ Center of Experimental Orthopaedics, Saarland University, Hamburg D-66421, Germany; ${ }^{4}$ Department of Sports Medicine and Arthroscopic Surgery, The First Affiliated Hospital of Anhui Medical University, Hefei, Anhui 230022, P.R. China
\end{abstract}

Received April 18,2020; Accepted November 9, 2020

DOI: $10.3892 / \mathrm{mmr} .2021 .11824$

\begin{abstract}
Stem cell-based therapy is a promising alternative to conventional approaches to treating intervertebral disc degeneration (IDD). However, comprehensive understanding of stem cell-based therapy at the gene level is still lacking. In the present study, we identified the expression profiles of messenger RNAs (mRNAs) and long non-coding RNAs (lncRNAs) expressed within a co-culture system of adipose-derived mesenchymal stem cells (ASCs) and degenerative nucleus pulposus cells (NPCs) and explored the signaling pathways involved and their regulatory networks. Microarray analysis was used to compare ASCs co-cultured with degenerative NPCs to ASCs cultured alone, and the underlying regulatory pattern, including the signaling pathways and competing endogenous RNA (ceRNA) network, was analyzed with robust bioinformatics methods. The results showed that 360 lncRNAs and 1757 mRNAs were differentially expressed by ASCs, and the microarray results were confirmed by quantitative PCR. Moreover, 589 Gene Ontology terms were upregulated, whereas 661 terms were downregulated. A total of 299 signaling pathways were significantly altered. A Path-net and a Signal-net were built to show interactions among differentially expressed genes. An mRNA-lncRNA co-expression network was constructed to reveal the interplay among differentially expressed mRNAs and IncRNAs, whereas a ceRNA network was built to investigate their connections with microRNAs involved in IDD. To the
\end{abstract}

Correspondence to: Dr Jianhong Wu, Trauma Centre, Department of Trauma and Orthopedics, Shanghai General Hospital, Shanghai Jiao Tong University, 650 Xin Songjiang Road, Shanghai 201620, P.R. China

E-mail: wujianhong1978@hotmail.com

Key words: gene microarray analysis, long non-coding RNA, microRNA, adipose-derived mesenchymal stem cell, intervertebral disc degeneration, nucleus pulposus cells best of our knowledge, this original and comprehensive exploration reveals differentially expressed lncRNAs and mRNAs of ASCs stimulated by degenerative NPCs, underscoring the regulation pattern within the co-culture system at the gene level. These data may further understanding of NPC-directed differentiation of ASCs and facilitate the application of ASCs in future treatments for IDD.

\section{Introduction}

Intervertebral disc degeneration (IDD), the leading cause of low back pain and spinal disabilities (1-3), poses a significant social and medical burden on adults worldwide. Various diseases secondary to IDD, namely, degenerative disc diseases (DDD), occur in approximately $71 \%$ of males and $77 \%$ of females older than age 50 and in more than $90 \%$ of the older population $(4,5)$.

Traditional surgical interventions that remove degenerative nucleus pulposus (NP) tissue and are dependent on internal fixation are not able to fully restore spinal function and may result in various complications, including deep infection and neurological impairment $(1,2,6-9)$. New stem cell-based therapies are promising alternatives to traditional interventions targeting early to middle-stage IDD before its irreversible progression requires open intervention. Specifically, stem cell-based therapies for IDD introduce exogenous or autologous mesenchymal stem cells (MSCs) into the target intervertebral disc via injection $(8,10-12)$. The rationale for this treatment is two-fold. First, MSCs can differentiate into NP-like cells, express NP marker genes (e.g., SOX9, type II collagen, and aggrecan), and stimulate the formation of the extracellular matrix (ECM), which partially enforces the biomechanical property of the disc and further protects the MSCs from the harsh intra-disc microenvironment. Second, the functioning of degenerative NPCs needs to be restored under the influence of cytokines and growth factors produced by the MSCs.

The potential of MSCs to treat disc degeneration has been proven in many in vitro $(6,8,13)$ and in vivo $(9-11)$ studies. Adipose-derived mesenchymal stem cells (ASCs) 
can be induced to differentiate into NP-like cells with significantly higher expression of specific markers, including aggrecan, SOX9, and type II collagen (14). Animal studies have confirmed such in vitro evidence and shown that ASCs injected into injured lumbar discs increase secretion of the ECM and decrease the ossification of damaged cartilage in the NP compared to untreated controls (15). Accumulating encouraging data on ASCs as well as their relatively ample availability and clinical safety make them one of the most promising candidates for stem cell therapy for IDD (16). However, the underlying cellular mechanisms of cross-talking between ASCs and degenerative target cells at the gene level have not yet been comprehensively investigated.

Long non-coding RNAs (lncRNAs) and microRNAs (miRNAs) have been increasingly investigated because of their regulatory functions in gene expression $(17,18)$. IncRNAs are involved in various biological processes with high tissue specificity and low sequence conservation $(19,20)$, and differentially expressed lncRNAs have previously been highlighted in the disc degeneration process $(21,22)$. Previous findings showed that IncRNAs were differentially expressed by degenerative nucleus pulposus cells (NPCs) when they were co-cultured with ASCs (22). Furthermore, miRNAs regulate gene expression, influence various biological activities [e.g., cell proliferation, differentiation, and metabolism (23)], and play noteworthy roles in the disc degeneration process (24-27). For instance, miR-27b (24) and miR-93 (25) specifically promote matrix degradation and hasten disc degeneration. However, to the best of our knowledge, no study has underlined the specific roles of lncRNAs and miRNAs within a co-culture of ASCs and degenerative NPCs that mimics the microenvironments of clinical ASCs in the treatment of IDD.

Advanced gene expression microarray and bioinformatics modalities were used to identify lncRNA and mRNA mappings during the differentiation of ASCs into NP-like cells and to further show specifically involved signaling pathways and gene regulation networks.

\section{Materials and methods}

Ethics approval and patient consent. All human tissue was obtained and used with patients' informed consent and under the approval of the institutional review broad of Shanghai General Hospital, Shanghai Jiao Tong University, Shanghai, China. A schematic of the study is shown in Fig. 1.

Isolation and culture of NPCs. The human NP tissue was obtained surgically from degenerative discs [grades III-IV according to the Pfirrmann grading system (12)] of three patients diagnosed with lumbar spondylosis. These patients (one female and two males, 46- to 58-years of age, with a mean age of 52) were enrolled and the tissue was collected at Shanghai General Hospital, Shanghai, China. NPCs were isolated according to a method previously reported $(6,8,12)$. Briefly, the NP tissue was stored in cold phosphate-buffered saline (PBS) and then transferred to the lab. After being washed in PBS to remove all blood and annulus fibrous tissue, the NP tissue was cut into $1 \mathrm{~mm}$ sections amid serum-free medium, i.e., Dulbecco's modified Eagle's medium/F12 medium (DMEM/F12; HyClone), supplemented with $1 \%$ penicillin/streptomycin. Then the tissue was digested with $0.4 \mathrm{mg} / \mathrm{ml}$ collagenase II (Sigma-Aldrich; Merck KGaA) at $37^{\circ} \mathrm{C}$ for $5 \mathrm{~h}$ before normal medium was used to end the process. Thereafter, the cell suspension was passed consecutively through filters $(100,70,40 \mu \mathrm{m})$ to remove debris, then centrifuged at $400 \mathrm{x} \mathrm{g}$ for $5 \mathrm{~min}$ in room temperature to form the cell pellets. The supernatant was discarded, and cells were resuspended and cultured in DMEM supplemented with $10 \%$ fetal bovine serum (FBS) in an incubator at $37^{\circ} \mathrm{C}$ with $5 \% \mathrm{CO}_{2}$ then passaged at $80 \%$ confluency. The medium was changed every 2-3 days.

Isolation, culture, and identification of ASCs. ASCs were identified and isolated from adipose tissue based on reported methods $(12,28,29)$. Briefly, adipose tissue was harvested from identical patients as the NP tissue, and then reserved in DMEM. After being carefully washed with PBS to remove blood, fat tissue was minced into $1 \mathrm{~mm}^{2}$ sections, and then treated with $0.1 \%$ type I collagenase (Sigma-Aldrich; Merck KGaA) at $37^{\circ} \mathrm{C}$ for $60 \mathrm{~min}$ on a shaking bench. Subsequently, the cell tissue mixture was filtered as mentioned previously, followed by centrifugation ( $400 \mathrm{x} \mathrm{g}$ for $5 \mathrm{~min}$ at room temperature) and removal of the supernatant. The cell pellets were resuspended with and cultured in DMEM/F12 (HyClone) containing 10\% FBS and $1 \%$ penicillin/streptomycin in an incubator at $37^{\circ} \mathrm{C}$ with $5 \% \mathrm{CO}_{2}$. The medium was changed every 2-3 days, and the cells were passaged when confluency reached $80 \%$.

Multilineage differentiation and flow cytometry were performed to identify the stemness of the isolated cells. Briefly, adipogenic medium was used to induce differentiation of candidate cells at passage 3. Adipogenic induction was performed by seeding cells in adipogenic medium supplemented with $0.5 \mathrm{mM}$ isobutyl methylxanthine, $0.5 \mathrm{mM}$ dexamethasone, and $60 \mathrm{mM}$ indomethacin (Sigma-Aldrich; Merck KGaA) and culturing them for 7 days. ASCs were cultured with osteogenic medium (STEMCELL) for 2 weeks, then fixed with $4 \%$ paraformaldehyde for subsequent staining; the chondrogenic potential of isolated cells was tested by seeding and culturing them with a specific chondrogenic medium for 14 days (STEMCELL) and then fixing them for evaluation. All culture media were changed every 2-3 days.

In vitro differentiation of ASCs was evaluated by histological staining for adipogenesis (Oil Red O staining), osteogenesis (Alizarin Red staining), and chondrogenesis (Alcian blue and type II collagen staining). Furthermore, expression of surface markers, including CD105, CD73, CD29, CD34, CD45, and human leukocyte antigen (HLA)-DR, was investigated as described previously (12).

Co-culture of ASCs and NPCs. To imitate stem cell therapy in vitro, we established a cell-cell co-culture system using a 6 -well plate with a $0.4 \mu \mathrm{m}$ pore size membrane. A total of $6.0 \times 10^{4}$ degenerative NPCs were seeded onto the base of the well, and the ASCs were seeded onto the upper surface of the membrane. Both NPCs and ASCs at passage 3 were used. The cell ratio was 1:1 and it was chosen based on the previous studies $(6,8)$. The cells were co-cultured for 7 days in $\mathrm{DMEM} / \mathrm{F} 12$ at $37^{\circ} \mathrm{C}$ and $5 \% \mathrm{CO}_{2}$ in a humidified atmosphere, and the medium was changed every 2 days. The co-cultured 


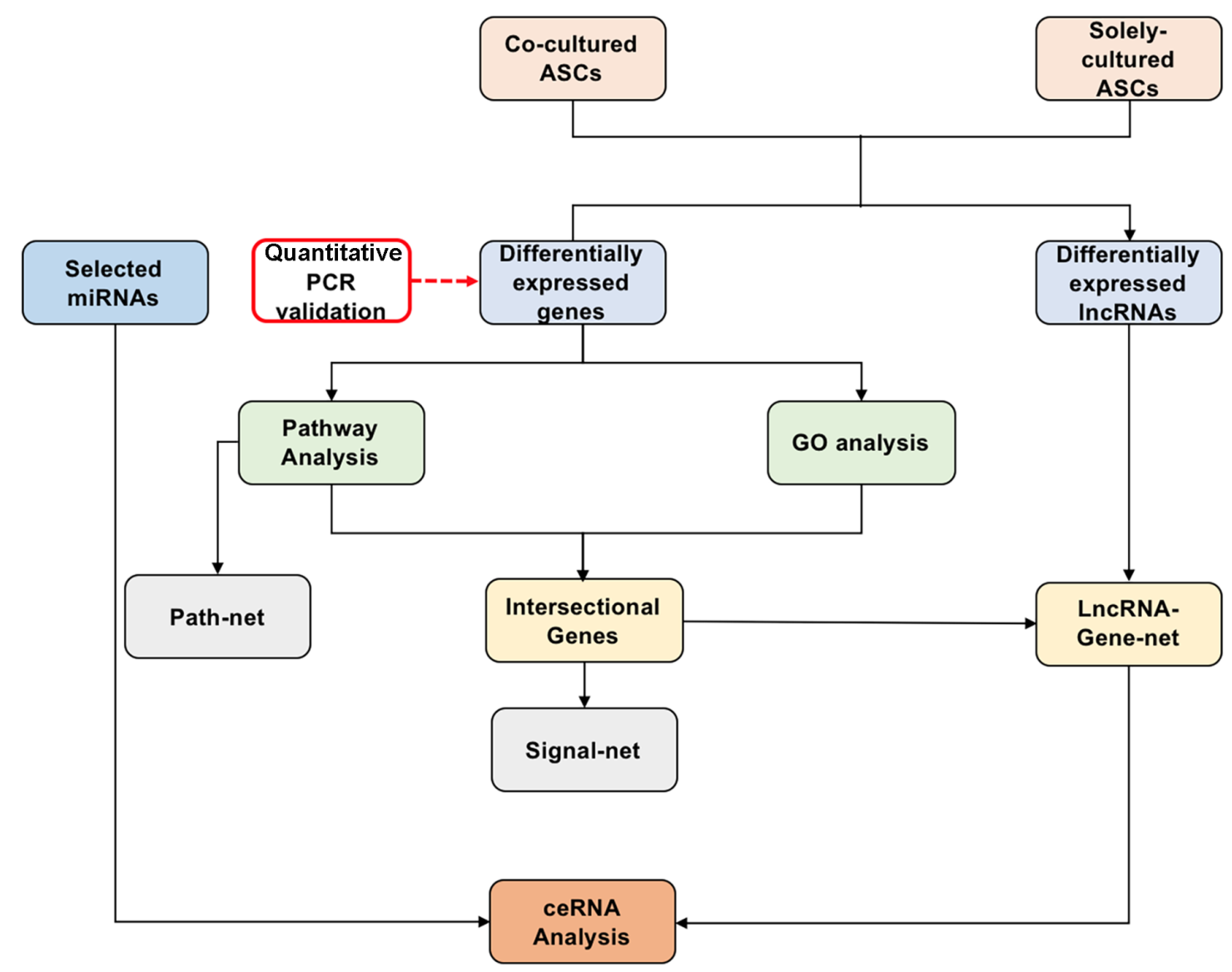

Figure 1. Diagram of the workflow of the present study, including cell culture, gene microarray assay, and bioinformatics analyses.

ASCs ( $n=3)$ formed the experimental group, whereas ASCs cultured alone in the same conditions $(n=3)$ acted as controls.

RNA isolation and quality control. Total RNA from co-cultured ASCs and ASCs was cultured alone using TRIzol (Invitrogen), then purified it with the RNase Kit (Bio-Rad Laboratories, Inc.). The quantity of RNA was measured using a spectrophotometer (NanoDrop-1000, Thermo Scientific Fisher) and tested its quality with $1 \%$ agarose gel electrophoresis as previously described (30).

Microarray analysis. A multistep strategy was used for the gene microarray and subsequent analyses (Fig. 1). ASCs co-cultured with degenerative NPCs (the experimental group) and ASCs cultured alone (the control group) were used to identify differentially expressed RNAs. Microarray analysis was performed by GMINIX Informatics Ltd. Co. (Shanghai, China). The data have been uploaded to the NCBI Gene Expression Omnibus and can be accessed via the GEO Series accession GSE118927 (ncbi.nlm.nih.gov/geo/query/acc.cgi?acc=GSE118927).

Differential expression of mRNAs and lncRNAs between the co-cultured ASCs and ASCs cultured alone was determined with a random variance model (RVM) t-test to increase the degrees of freedom in the small data sets. $\mathrm{P}<0.05$ as the threshold for significance, these differentially expressed RNAs were further selected and evaluated with false discovery rate analysis. Unsupervised hierarchical clustering was performed to generate a cluster map.
Quantitative (q)PCR validation. To verify the microarray data, six RNAs were randomly selected for qPCR validation. Briefly, cDNA was generated with the Taqman Reverse Transcription kit (Invitrogen; Thermo Fisher Scientific, Inc.) following the manufacturer's protocol. Gene expression was investigated by qPCR with the SYBR-Green Mastermix on a CFX96 Touch Real-time PCR Detection System (both Bio-Rad Laboratories, Inc.). The thermocycling conditions (totally 35 cycles) consisted of the initial denaturation of $30 \mathrm{sec}$ at $95^{\circ} \mathrm{C}$, followed by $60 \mathrm{sec}$ annealing at $65^{\circ} \mathrm{C}$, and the extension step lasted for $1 \mathrm{~min} / \mathrm{kb}$ at $68^{\circ} \mathrm{C}$. Human GAPDH was used as the housekeeping gene to determine the relative expression of selected genes, and relative changes in gene expression were compared to those of untreated cells by the $2^{-\Delta \Delta \mathrm{Cq}}$ method (12). All reactions were performed in triplicate, and the primer sequences are provided in Table I.

Gene ontology $(G O)$ analysis. Using the GO Database (GO Consortium, geneontology.org), we performed GO analysis to explore the functions of the differentially expressed mRNAs using the two-sided Fisher's exact test and Chi-square test. Each differentially expressed gene was evaluated independently and marked as up- or downregulated. P-values of all differentially expressed genes were computed in all GO categories, and $\mathrm{P}<0.01$ was considered to be significant.

Pathway analysis. The significance levels of pathways associated with differentially expressed genes were analyzed based on the Kyoto Encyclopedia of Genes and Genomes 
Table I. Primers used in qPCR.

Genes

\section{IGF1}

XIST

TTTY15

FABP3

TGFB2

GPC3

GAPDH
Forward sequence 5'-3'

Reverse sequence 5'-3'

GCTCTTCAGTTCGTGTGTGGA
TCTAGTCCCCAACACCCTT
CTCATCACCTGGAGTCCGTG
TGGAGTTCGATGAGACAACAGC
CAGCACACTCGATATGGACCA
ATTGGCAAGTTATGTGCCCAT
GGAGCGAGATCCCTCCAAAAT

GCCTCCTTAGATCACAGCTCC GGAGGACGTGTCAAGAAGACA CAACGGCAAATACTTTAGGTTTTCT CTCTTGCCCGTCCCATTTCTG CCTCGGGCTCAGGATAGTCT TTCGGCTGGATAAGGTTTCTTC GGCTGTTGTCATACTTCTCATGG

IGF1, insulin-like growth factor 1; XIST, X inactive-specific transcript; TTTY1, testis-specific transcript Y-linked 15; FABP3, fatty acid binding protein 3; TGFB2, transforming growth factor, beta 2; GPC3, glypican 3; GAPDH, glyceraldehyde 3-phosphate dehydrogenase.

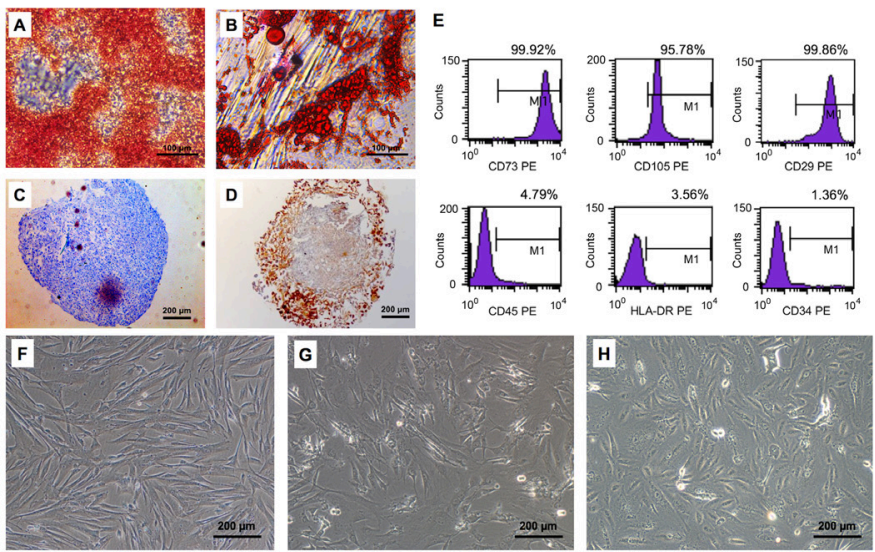

Figure 2. Characterization of ASCs by multilineage differentiation. Osteogenic (A) and adipogenic (B) differentiation is quantified by Alizarin Red S and Oil Red O staining, respectively. Chondrogenic differentiation from ASCs is evidenced by Alcian blue staining (C) and immunohistochemistry (D) of type II collagen. Surface markers of ASCs (E) are positive for CD73, CD105, and CD29 and negative for CD34, CD45, and HLA-DR. (F) Solely cultured ASCs at day 7 . ASCs $(\mathrm{G})$ before and $(\mathrm{H})$ after co-culturing with NPCs. After 7 days of co-culturing with NPCs, ASCs became relatively round and small as NP-like cells.

A

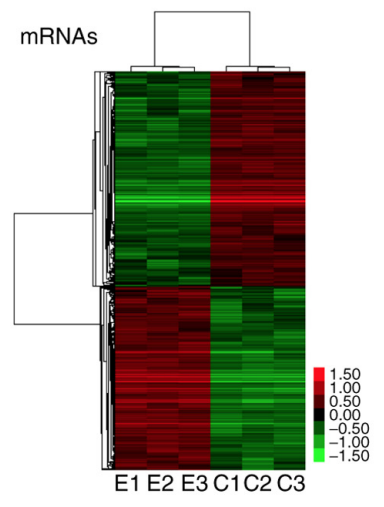

B

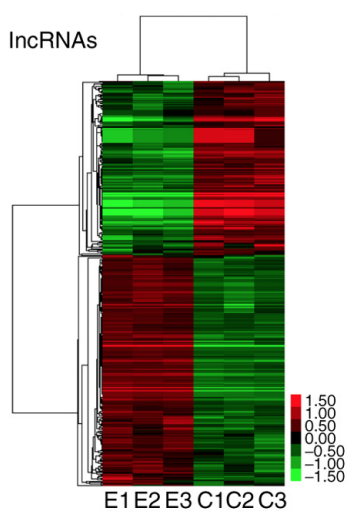

Figure 3. Clustering heat map of differentially expressed mRNAs and lncRNAs. The $\mathrm{x}$ and $\mathrm{y}$ axes indicate the sample groups and probes, respectively. (A) A total of 1,757 genes and 360 lncRNAs were differentially expressed by ASCs under the influence of NPCs. Carboxypeptidase X (M14 family) member 1 (CPXM1) was the most upregulated gene (28-fold change; $\mathrm{P}<0.001)$, and mRNA coding cartilage oligomeric matrix protein $(\mathrm{COMP})$ was the most downregulated gene $(-61$-fold change; $\mathrm{P}<0.001)$. (B) The expression of 360 lncRNAs were significantly altered, among which XIST (X-inactive specific transcript) was most upregulated (23-fold change), and SNAR-E was most downregulated (12-fold change). The red stripe shows high relative expression, and the green stripe shows low relative expression. E, experimental group of ASCs co-cultured with degenerated NPCs; C, control group of ASCs cultured alone.

database (KEGG; genome.jp/kegg/). Fisher's exact test and the Chi-square test were used to select the key pathways according to a significance threshold of $\mathrm{P}<0.05$. Additionally, a Path-net, the interaction network consisting of the significant pathways, was generated based on information for those pathways from the KEGG database.

Signal-net analysis. Using the KEGG database, we constructed a Signal-net to reveal the gene-gene interplay among the significant differentially expressed genes in both the GO analysis and pathway analysis. The Signal-net shows molecular networks consisting of nodes and lines representing genes and regulatory patterns of activation or phosphorylation. We calculated the importance of each gene by counting the numbers of upstream and downstream genes in the form of in-degrees and out-degrees. The betweenness centrality of each gene was recorded according to its in-degree and out-degree; a higher betweenness centrality suggesting more importance in the regulation of the gene network.

lncRNA-mRNA-net analysis. The co-expression network of lncRNAs and mRNAs was investigated based on differentially expressed lncRNAs and intersectional mRNAs with both GO analysis and pathway analysis. 
A

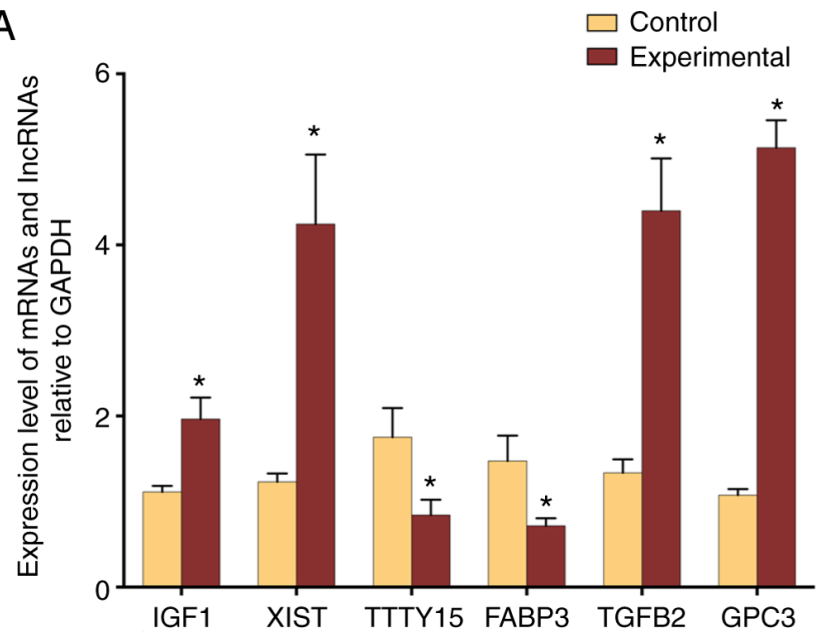

B

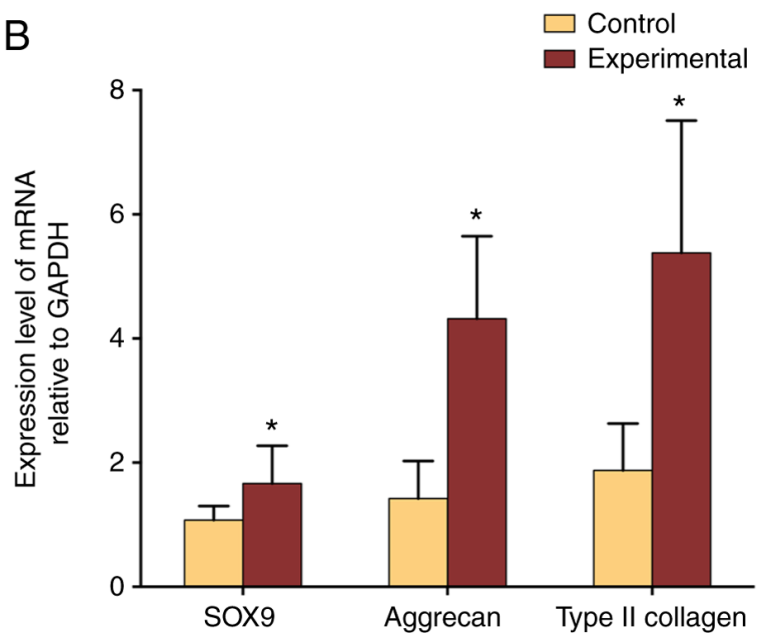

Figure 4. Validation of microarray data with real-time PCR. (A) Expression of IGF1, XIST, TGFB2, and GPC3 was significantly upregulated in the experimental group of ASCs co-cultured with degenerated NPCs compared to control ASCs cultured alone, whereas the expression of TTTY15 and FABP3 was significantly downregulated in the experimental group. (B) Expression levels of NPC specific maker genes. The expression of SOX9, type II collagen, and aggrecan were significantly higher in the Experimental group than these in the Control group $(\mathrm{P}=0.0153, \mathrm{P}=0.0003, \mathrm{P}<0.0001$, respectively). "P<0.05. IGF1, insulin-like growth factor 1; XIST, X inactive-specific transcript; TTTY15, testis specific transcript, Y-linked 15; FABP3, fatty acid binding protein 3; TGFB2, transforming growth factor, beta 2; GPC3, glypican-3.

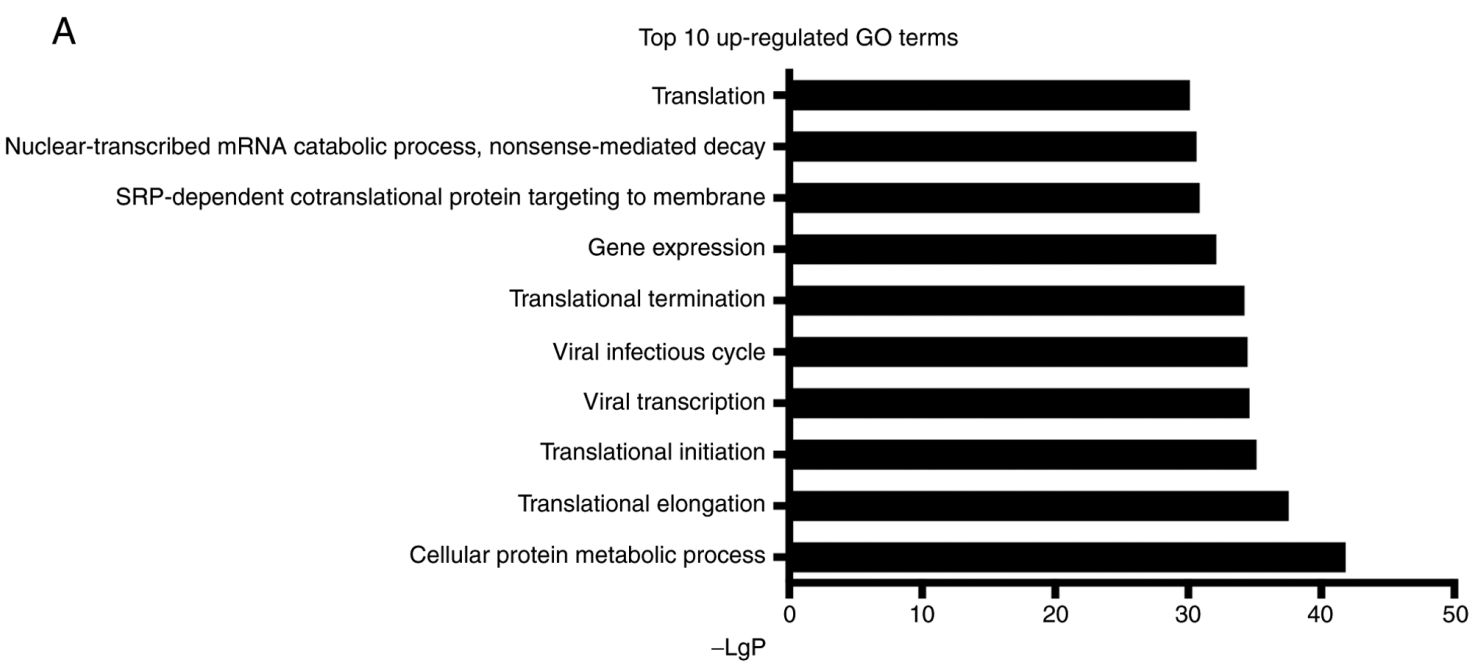

B

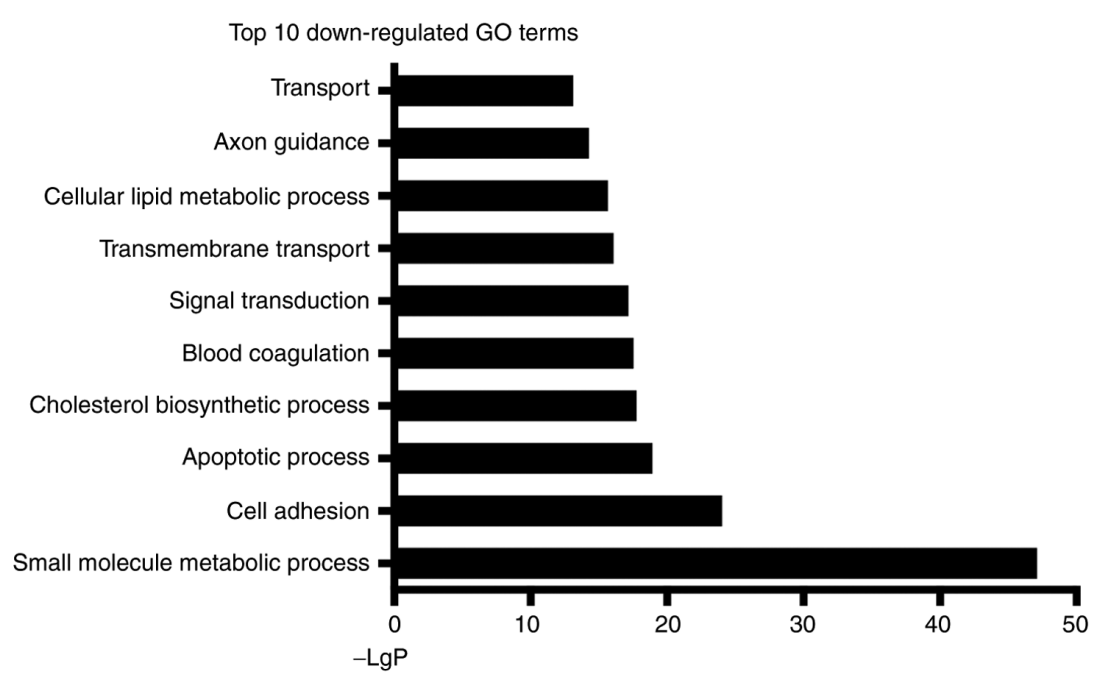

Figure 5. Top 10 most significantly (A) up- and (B) downregulated GO terms during the co-culture of ASCs and degenerative NPCs. The cellular protein metabolic process (GO:0044267) was the most upregulated ( $\mathrm{LgP}=41.8) \mathrm{GO}$ term, and the small molecule metabolic process (GO:0044281) was the most downregulated $(-\mathrm{LgP}=47.1)$. The length of the bars on the $\mathrm{x}$ axis represents the negative logarithm of the $\mathrm{P}$-value $(-\mathrm{LgP})$ of each $\mathrm{GO}$ term, and $-\mathrm{LgP}$ is significantly positively correlated with the regulation of specific GO terms. 
A

Top 10 up-regulated pathways

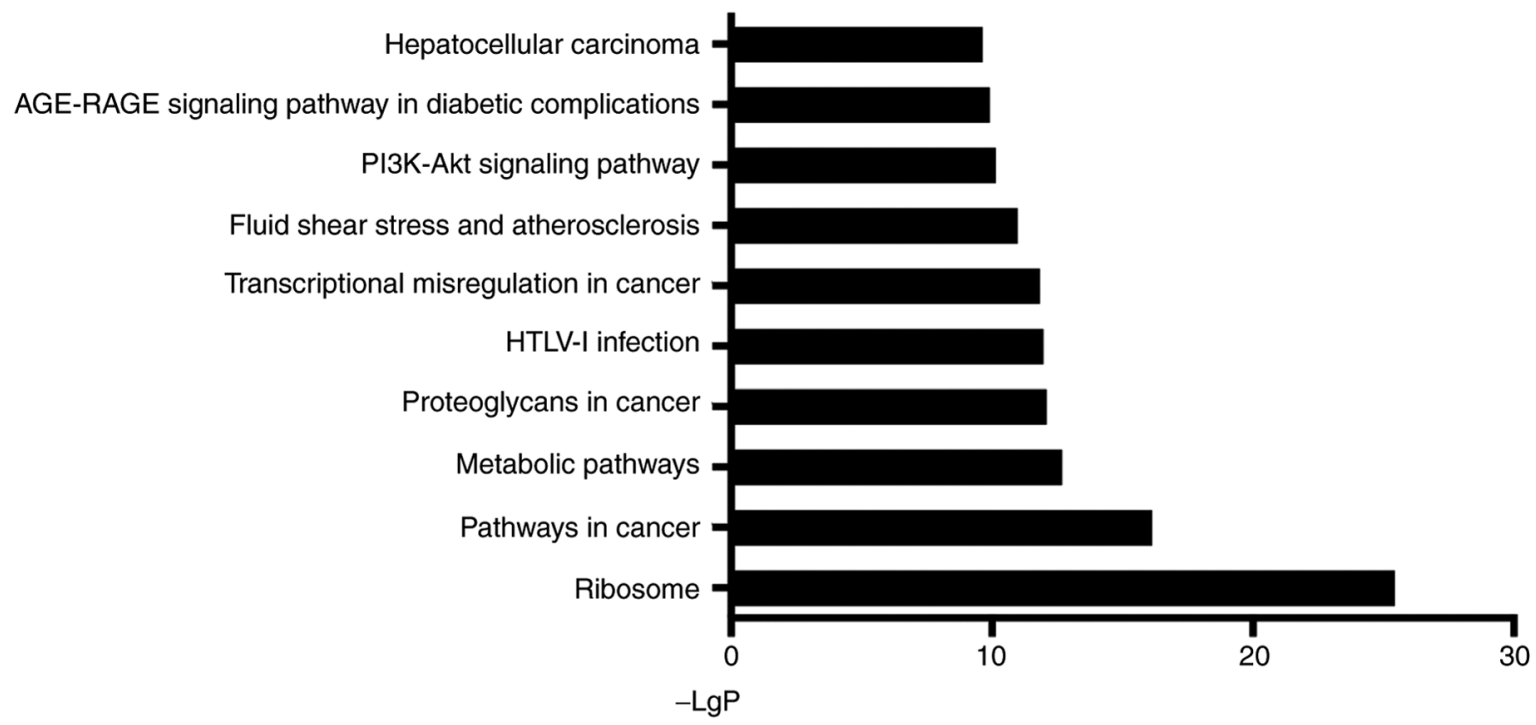

B

Top 10 down-regulated pathways

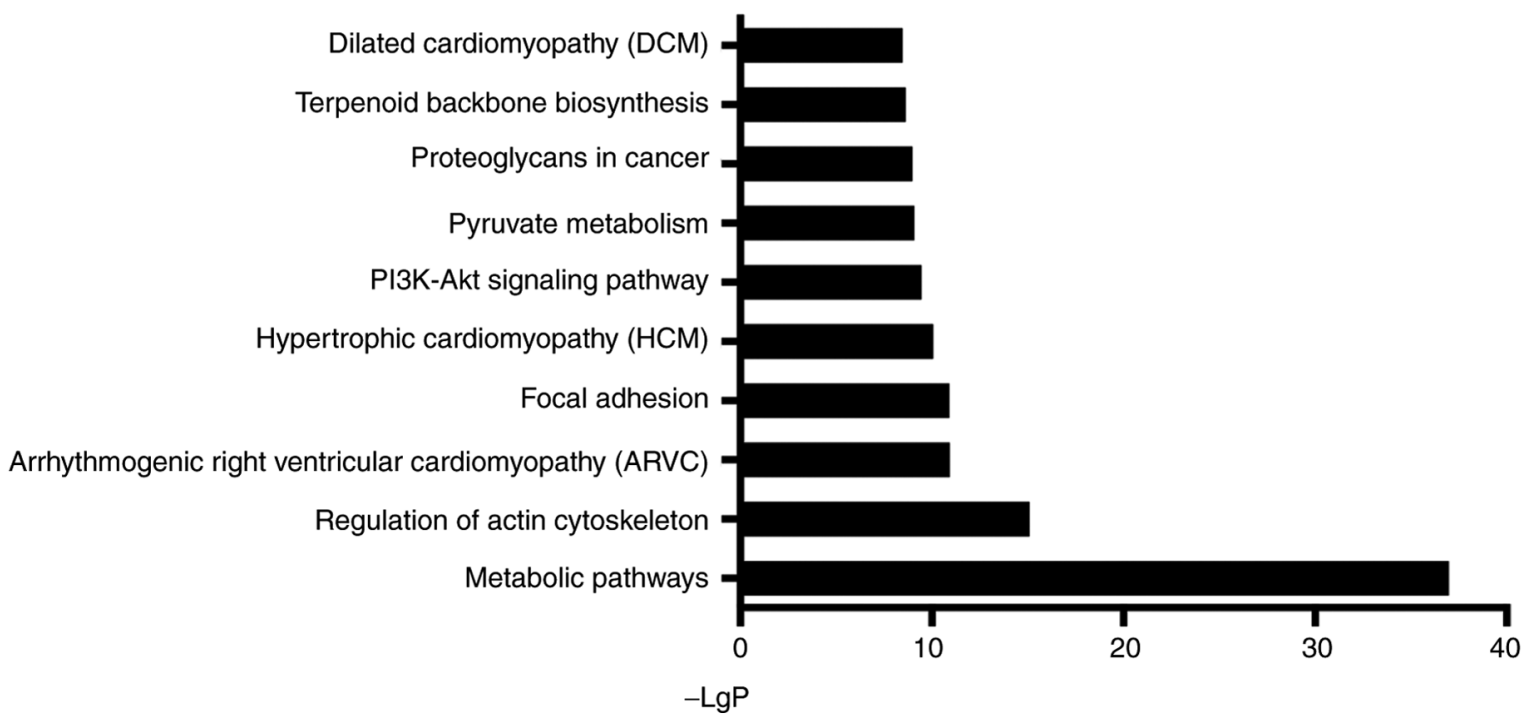

Figure 6. KEGG pathway analysis identifying the 10 most significantly upregulated (A) and downregulated (B) pathways during the co-culture of ASCs and degenerative NPCs. A total of 299 pathways were significantly enriched, of which 144 pathways were upregulated and 155 were downregulated (all P<0.05). In particular, ribosome (Path ID: 03010) was the most upregulated (-LgP=25.4), whereas the metabolic pathways (Path ID: 01100) were the most downregulated $(-\operatorname{LgP}=37.0)$.

Competing endogenous RNA (ceRNA) analysis. miRNAs are single-strand non-coding RNAs shorter than 22 nucleotides responsible for regulating gene expression by binding to miRNA response elements (MREs) on mRNAs $(31,32)$. lncRNAs are also non-coding RNAs but are longer than 200 nucleotides; they both harbor MREs and compete with RNAs for miRNA binding. IncRNAs regulate the number of miRNA by sequestering and binding to them (33); thus, they can participate in post-transcriptional regulation as ceRNAs.

We selected 25 miRNAs associated with disc degeneration that were differentially expressed by ASCs co-cultured with degenerative NPCs and investigated their roles in the regulation of mRNAs and lncRNAs (Table SI). The target mRNAs of miRNAs were predicted based on TargetScan (www.targetscan. org/) and miRanda (www.microrna.org/microrna/home.do) for the ceRNAs analysis. The ceRNA network was further constructed by those negatively regulated intersectional lncRNAs and mRNAs.

Statistical analysis. Data are presented as the mean \pm SD. Differences between groups were evaluated with unpaired Student's t-test in SPSS 20.0 (IBM). Statistical significance was set at $\mathrm{P}<0.05$.

\section{Results}

Multilineage differentiation and identification of ASCs. The cells isolated from the human adipose tissue were demonstrated to be MSCs with the stemness of multilineage differentiation (Fig. 2A-D) and mesenchymal markers (Fig. 2E). In particular, 


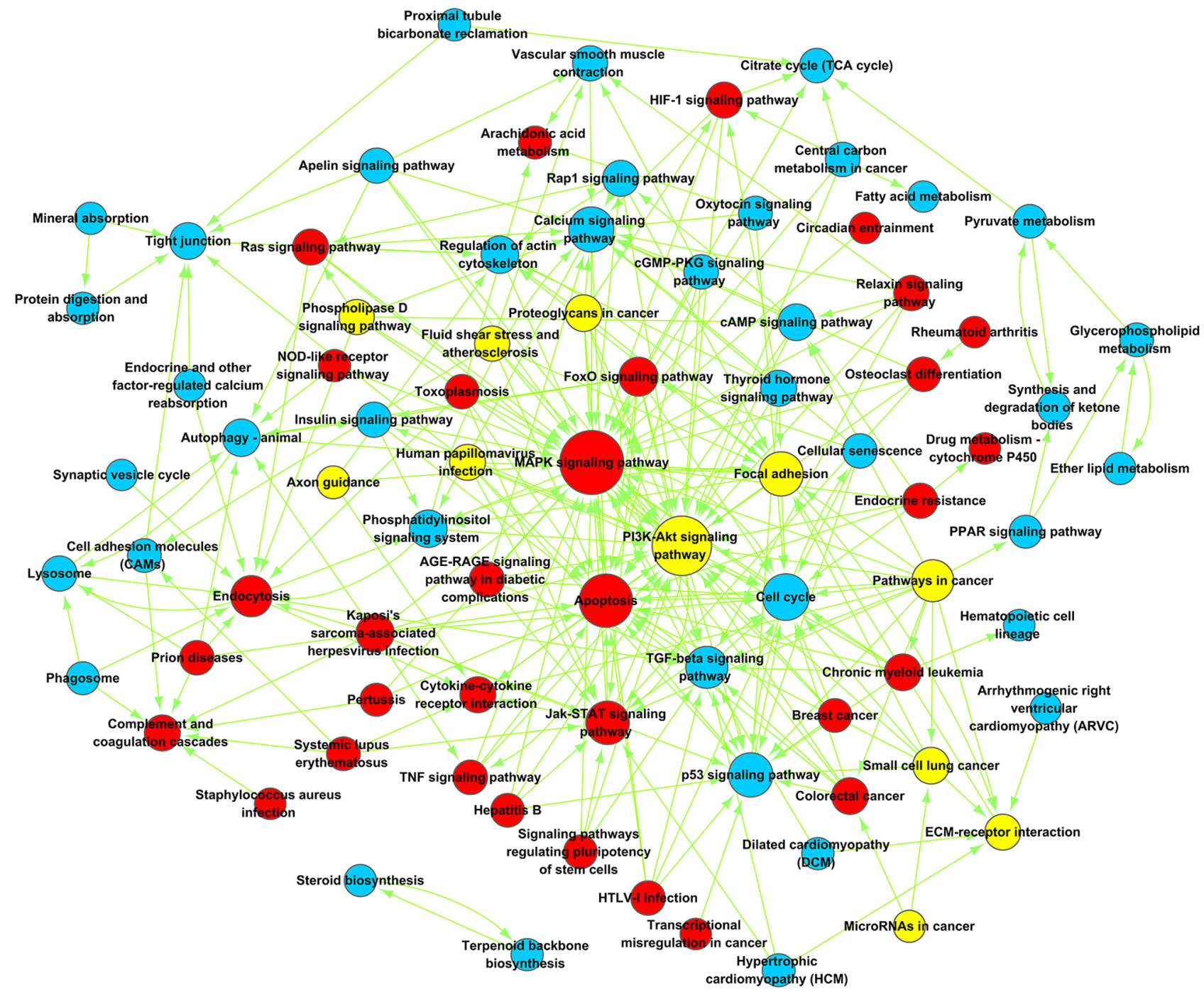

Figure 7. Path-net characterizing interactions among pathways. The MAPK signaling pathway was upregulated with the highest betweenness centrality at 44 , and the PI3K-AKT signaling pathway had the second highest betweenness centrality (degree=38), as it contained both up- and downregulated genes. Up- and downregulated pathways are highlighted in red and blue, respectively, and pathways with both up- and downregulated genes are indicated in yellow. The circle size represents the number of other pathways interacting with the specific pathway (i.e., the degree of the pathway).

the ASCs differentiated into osteocytes and adipose cells as shown by positive Alizarin Red (Fig. 2A) and Oil Red O (Fig. 2B) staining, respectively. In addition, the ASCs differentiated into chondrocytes (Fig. 2C) and produced type II collagen in the chondrogenic environment (Fig. 2D). Furthermore, MSC surface markers expressed by ASCs included CD73, CD105, and CD29; no hematopoietic stem cell markers (e.g. CD45, CD34 or HLA-DR) were detected (Fig. 2E).

Differentially expressed mRNAs and IncRNAs in co-cultured ASCs. A total of 1757 genes were found to be differentially expressed by ASCs, of which carboxypeptidase X (M14 family) member 1 (CPXM1) had the largest increase in expression $(28$-fold change; $\mathrm{P}<0.001)$ and mRNA coding cartilage oligomeric matrix protein (COMP) had the largest decrease in expression (-61-fold change; $\mathrm{P}<0.001$; Fig. 3A).

A total of $360 \mathrm{lncRNAs}$ were identified as differentially expressed by ASCs, of which XIST (X-inactive specific transcript) was maximally upregulated (23-fold change) and SNAR-E was greatly downregulated (12-fold change; Fig. 3B).
qPCR validation. The microarray data were further validated through six differentially expressed RNAs (IGF1, XIST, TTTY15, FABP3, TGFB2, GPC3) that were randomly selected for qPCR analysis. Of these RNAs, upregulation of IGF1, XIST, TGFB2, and GPC3 in the microarray was validated (all $\mathrm{P}<0.05$; Fig. 4). Similarly, in good agreement with gene-chip data, TTTY15 and FABP3 were demonstrated to be downregulated (both $\mathrm{P}<0.05$; Fig. $4 \mathrm{~A}$ ). In addition, the expression levels of SOX9, type II collagen, and aggrecan were significantly higher in the Experimental group than these in the Control group (all $\mathrm{P}<0.05$, Fig. 4B).

$G O$ analysis. The GO analysis identified 589 upregulated and 661 downregulated GO terms among all differentially expressed mRNAs. In particular, cellular protein metabolic process (GO:0044267) was most significantly upregulated (-LgP=41.8; Fig. 5), and the small molecule metabolic process (GO:0044281) was most considerably downregulated $(-\mathrm{LgP}=47.1)$. Note that various $\mathrm{GO}$ terms related to gene translation could be observed among the 10 most upregulated 
Table II. Top 10 pathways in path-net analysis.

\begin{tabular}{llccc}
\hline Path name & Style & Degree & Indegree & Outdegree \\
\hline MAPK signaling pathway & Up & 44 & 40 & 4 \\
PI3K-Akt signaling pathway & Up, down & 38 & 30 & 8 \\
Apoptosis & Up & 30 & 25 & 5 \\
Cell cycle & Down & 21 & 19 & 2 \\
Calcium signaling pathway & Down & 19 & 16 & 3 \\
Focal adhesion & Down, up & 18 & 11 & 7 \\
p53 signaling pathway & Down & 18 & 15 & 3 \\
Jak-STAT signaling pathway & Up & 17 & 12 & 5 \\
Pathways in cancer & Up, down & 15 & 0 & 15 \\
TGF-beta signaling pathway & Down & 15 & 12 & 3 \\
\hline
\end{tabular}

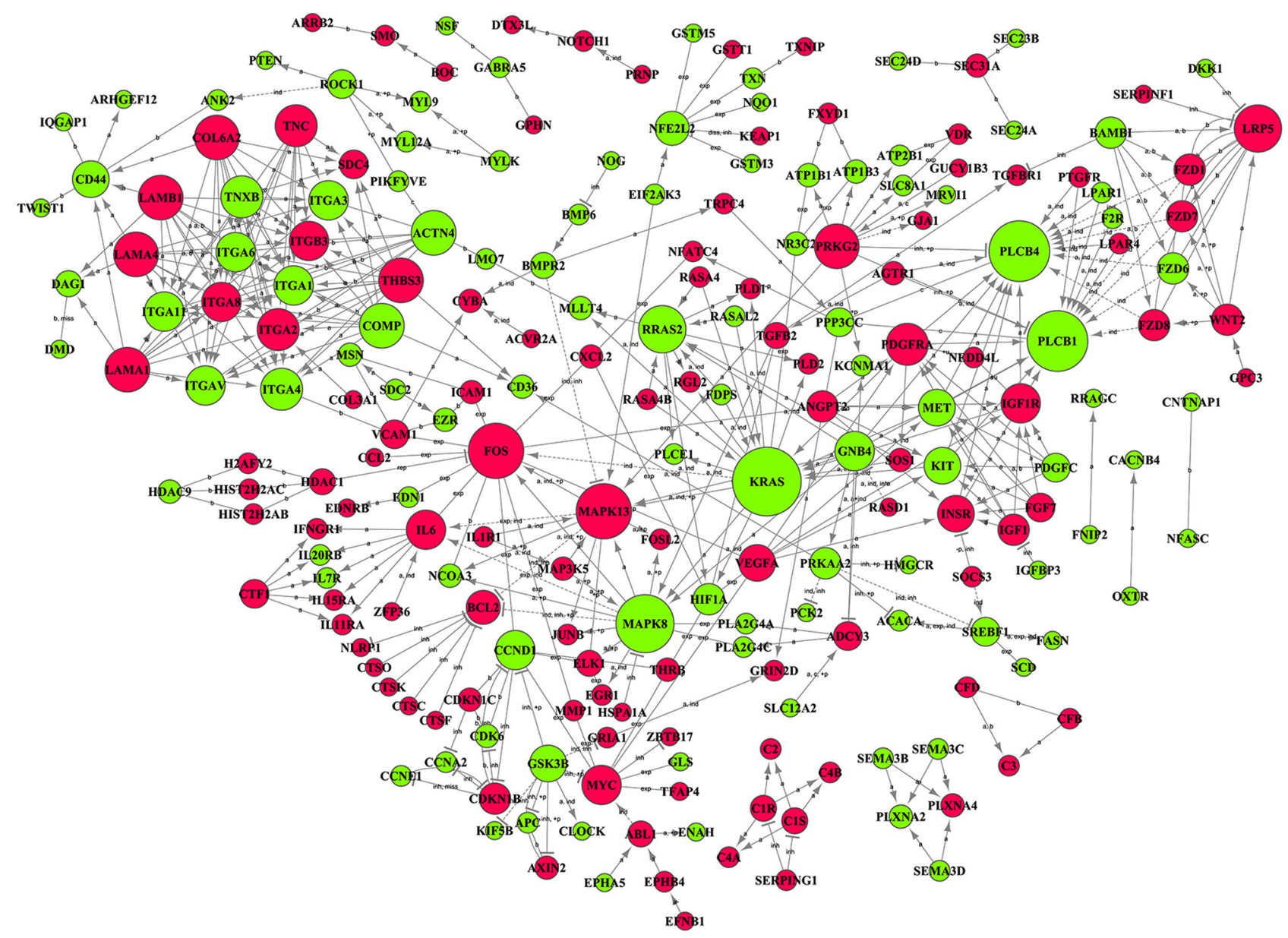

Figure 8. Signal-net showing the interaction among 218 differentially expressed genes. The KRAS gene, which was downregulated, was the most critical player in the Signal-net (degree=20), and FOS was the most important upregulated gene in the network (degree $=15)$. Up- and downregulated genes are indicated by red and green circles, respectively. The circle size represents the number of other genes interacting with the specific gene. Inter-gene interactions are reported as activation (a), binding/association (b), compound (c), phosphorylation (+p), repression (rep), ubiquitination (+u), inhibition (inb), expression (exp), dephosphorylation (-p), indirect effect (ind), missing interaction (miss), and dissociation (diss).

terms, including translational elongation (GO:0006414), translational initiation (GO:0006413), and translational termination (GO:0006415).

Pathway analysis. A total of 299 pathways were identified as significantly enriched during the co-culture of ASCs and degenerative NPCs, of which 144 pathways were significantly upregulated and 155 were significantly downregulated (all $\mathrm{P}<0.05$ ). Specifically, ribosome (Path ID: 03010) was the most upregulated, with a $-\mathrm{LgP}$ of 25.4 , whereas the metabolic pathways (Path ID: 01100) were the most significantly downregulated, with a -LgP of 37.0 (Fig. 6). 
Table III. Top 10 genes in Signal-Net analysis.

\begin{tabular}{llccc}
\hline Gene symbol & Style & Degree & Indegree & Outdegree \\
\hline KRAS & Down & 20 & 11 & 9 \\
PLCB1 & Down & 17 & 16 & 1 \\
PLCB4 & Down & 17 & 16 & 1 \\
MAPK8 & Down & 16 & 5 & 11 \\
FOS & Up & 15 & 5 & 10 \\
MAPK13 & Up & 15 & 8 & 7 \\
LRP5 & Up & 12 & 8 & 4 \\
RRAS2 & Down & 12 & 6 & 6 \\
ACTN4 & Down & 11 & 1 & 10 \\
COL6A2 & Up & 11 & 0 & 11
\end{tabular}

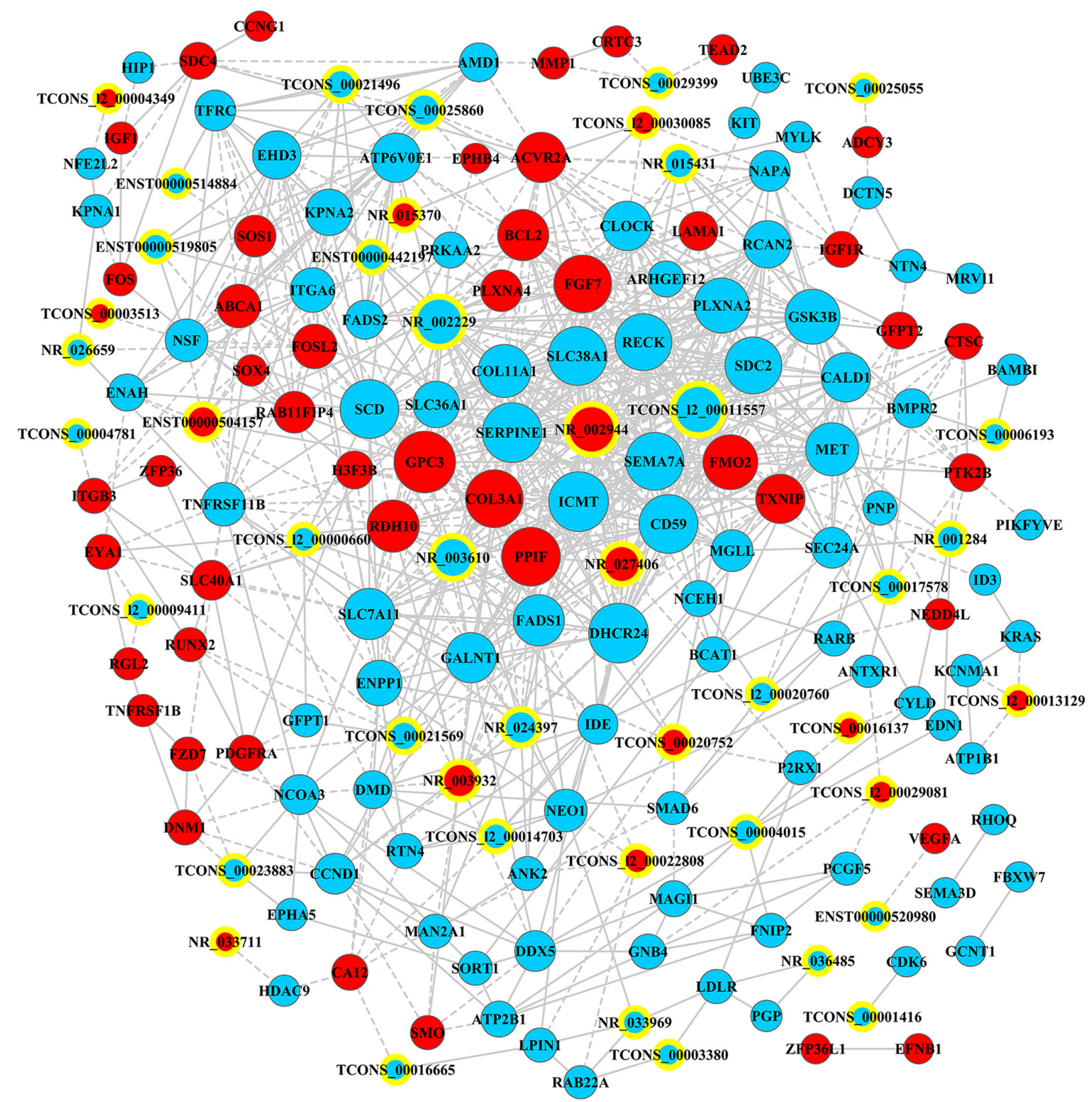

Figure 9. IncRNA-mRNA co-expression network of 44 lncRNAs (yellow ring nodes) and 134 mRNAs (circles); a total of 875 pairs of co-expressed lncRNA-mRNA were identified. In the co-expression network, GPC3 (glypican-3; coding; downregulated) had the highest number of interactions (degree=34), whereas TCONS_12_00011557 was a non-coding RNA that had the highest degree of interaction. Positive and negative interactions are shown as solid and dashed lines, respectively. Up- and downregulated RNAs are highlighted in red and blue, respectively. 
Table IV. Top 10 mRNAs and lncRNAs in lncRNA-mRNA co-expression network.

\begin{tabular}{lllc}
\hline Gene symbol & Biotype & Style & Degree \\
\hline GPC3 & Coding & Up & 34 \\
SERPINE1 & Coding & Down & 32 \\
ICMT & Coding & Down & 32 \\
DHCR24 & Coding & Down & 32 \\
SLC38A1 & Coding & Down & 31 \\
PPIF & Coding & Up & 31 \\
CD59 & Coding & Down & 31 \\
SCD & Coding & Down & 31 \\
FGF7 & Coding & Up & 30 \\
SEMA7A & Coding & Down & 30 \\
TCONS_12_00011557 & Noncoding & Down & 29 \\
NR_002229 & Noncoding & Down & 28 \\
NR_002944 & Noncoding & Up & 28 \\
NR_003610 & Noncoding & Down & 20 \\
NR_027406 & Noncoding & Up & 17 \\
NR_003932 & Noncoding & Up & 14 \\
NR_024397 & Noncoding & Down & 13 \\
ENST00000504157 & Noncoding & Up & 12 \\
TCONS_00025860 & Noncoding & Down & 12 \\
NR_015431 & Noncoding & Down & 10 \\
\hline
\end{tabular}

The Path-net was constructed to elucidate the complex interactions among all significantly enriched pathways and to represent all directly and systematically involved pathways during the co-culture of ASCs with NPCs (Fig. 7; Table II). The mitogen-activated protein kinase (MAPK) signaling pathway was found to play an important role in the differentiation of ASCs into NP-like cells, with the highest betweenness centrality at 44 . The PI3K-AKT signaling pathway was both up- and downregulated during differentiation with the second highest betweenness centrality (degree $=38$ ). The cell cycle pathway was the most significantly downregulated signaling pathway.

Signal-net. The Signal-net was constructed to explore the interactions of all genes with significant alterations in both the GO and pathway analyses (Fig. 8, Table III). The KRAS gene was found to play the most important role (downregulated; degree $=20$ ) within the differentiation of ASCs into NP-like cells, followed by phospholipase C $\beta-1$ (PLCB1) and PLCB4 (both downregulated; degree=17). By contrast, FOS showed the most significant upregulation (degree=15). In good agreement with the results of the pathway analysis, MAPK family genes were also identified as playing critical roles in the Signal-net, including MAPK8 (downregulated; degree=16) and MAPK13 (upregulated; degree=15).

IncRNA-mRNA co-expression network. A IncRNA-mRNA co-expression network was established on the basis of 178 RNAs (44 lncRNAs and 134 mRNAs) converting into 875 pairs of co-expressed lncRNA-mRNA (Fig. 9; Table IV). In the co-expression network, GPC3 (glypican-3; coding; downregulated; degree $=34$ ) had the highest number of interactions, followed by SERPINE1 (serpin peptidase inhibitor, clade E, member 1; coding; downregulated; degree $=32$ ) and ICMT (isoprenylcysteine carboxyl methyltransferase; coding; downregulated; degree=32), whereas TCONS_12_00011557 had the highest interaction (degree $=29$ ) among all non-coding RNAs.

ceRNA network. A ceRNA network was constructed from 25 IDD-related miRNAs to investigate their regulatory interplay with differentially expressed mRNAs and lncRNAs during the differentiation of ASCs into NP-like cells (Fig. 10; Table V). It is noteworthy that hsa-miR-27b-3p (degree of interaction=50) was the most important miRNA, followed by hsa-miR-93-5p (degree of interaction $=43$ ) and hsa-miR-27a-3p (degree of interaction=30). NR_002229, NR_003610, and NR_024397 were the three most important lncRNAs in the regulatory network, with degrees of interaction of 19,16 , and 11 , respectively.

\section{Discussion}

We identified 1,757 differentially expressed genes and 360 differentially expressed lncRNAs in ASCs co-cultured with degenerative NPCs. Among all the differentially expressed genes, 559 significantly upregulated and 661 significantly downregulated GO terms were identified involving 298 significantly enriched pathways. These results highlight the sophisticated interplay of these mRNAs and lncRNAs with miRNAs.

The MAPK and PI3K-Akt signaling pathways were identified as playing crucial roles within NPC-directed differentiation of ASCs. Three distinct MAPK subsets have been identified in mammalian cells: Classical MAPK (ERK), p38 kinase, and C-Jun N-terminal kinase/stress-activated protein kinase (JNK/SAPK) (34). In general, the MAPK signaling pathway is involved in many complex cellular processes, including proliferation, differentiation, development, and apoptosis. It can convey, amplify, and integrate external bio-signals and elicit cellular responses (34). Regarding IDD, the MAPK signaling pathway participates in many pathophysiological mechanisms and contributes to disc degeneration $(35,36)$. Tian et al reported the increasing degradation of aggrecan with modulated expression of ADAMTS-4 (A dis-integrin and metalloproteinase with thrombospondin motifs 4) in NPCs through activation of MAPK and NF- $\mathrm{BB}$ signaling (37).

However, our data indicate that the MAPK signaling pathway was upregulated when the ASCs differentiated into NP-like cells in vitro, which suggests that this pathway is also important in the regeneration of a degenerated disc. Such a discrepancy between previous studies and the present one is possibly attributable to the diverse roles this signaling pathway plays in various cellular behaviors, including both metabolic (e.g., proliferation and differentiation) and catabolic (e.g., apoptosis) activity. Similar positive roles of the MAPK signaling pathway have been previously reported. For instance, the p38 MAPK signaling pathway is activated after estrogen-induced enhancement of matrix synthesis in NPCs, whereas inhibiting p38 MAPK signaling significantly abolishes such effects of estrogen on matrix synthesis in NPCs (38). 


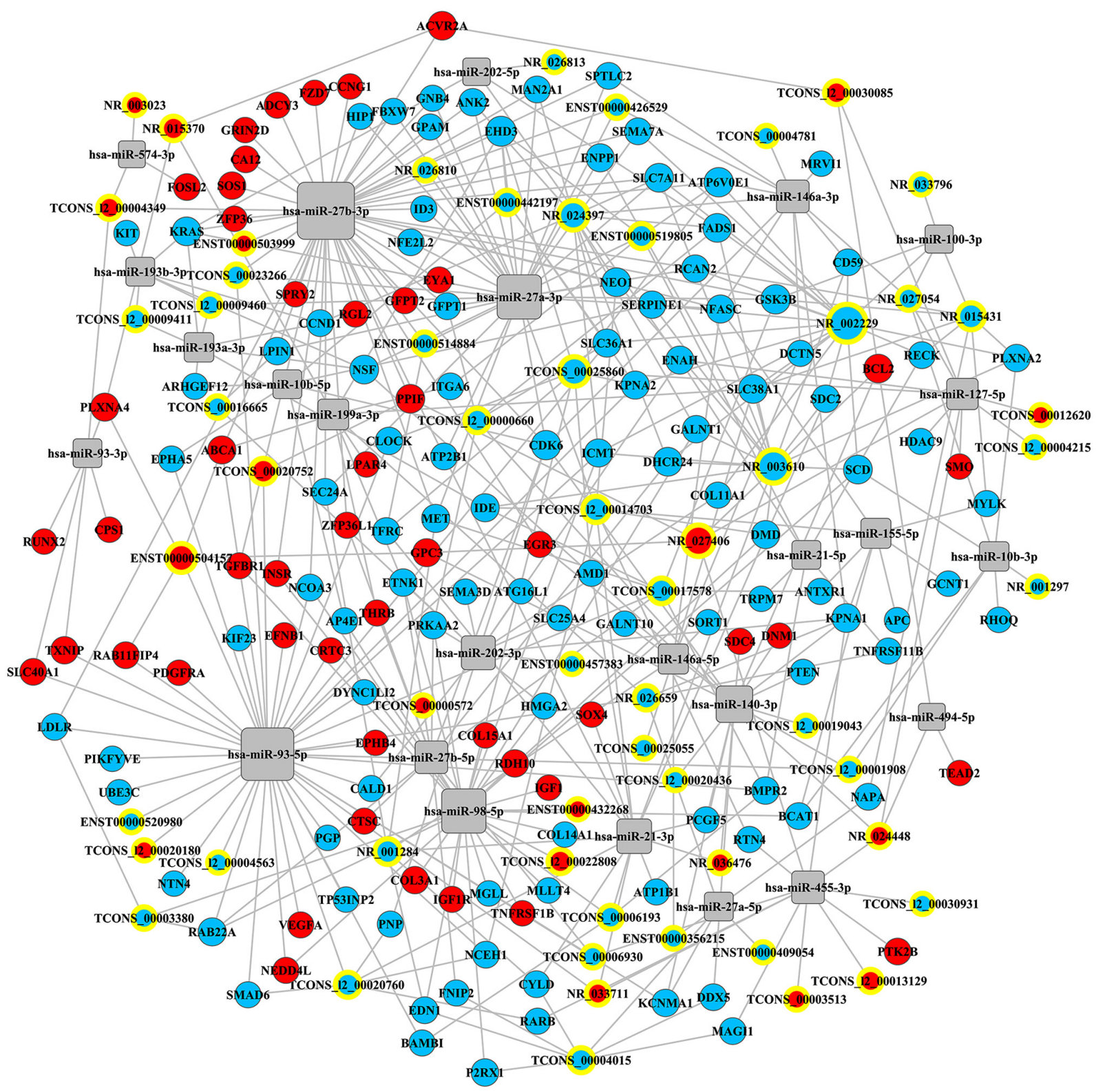

Figure 10. ceRNA network among 25 miRNAs, 152 mRNAs, and 58 lncRNAs. hsa-miR-27b-3 had the highest betweenness centrality at 50 . In addition, NR_002229 was the lncRNA in this regulatory network with the largest degree of interaction at 19. Upregulated RNAs are shown in red, and downregulated RNAs are shown in blue. miRNAs, mRNA, and lncRNAs are designated by rounded rectangles, circles, and yellow ring nodes, respectively.

The most important finding of the Signal-net analysis is that the COMP gene was closely associated with integrins (ITG) family members, including ITGB3, ITGA2, and ITGA3. COMP can interact with ECM proteins, bridging those molecules in a mesh pattern (39). Specifically, it binds to type II collagen in the presence of $\mathrm{Zn}^{2+}$ at four defined sites on the collagen molecule (40), and it also connects to aggrecan in a concentration-dependent manner (41). The present study emphasizes a tight correlation between COMP and the ITG family, which is partially consistent with findings from Chen et al, where COMP mediates chondrocyte attachment through its interactions with integrins (42). In addition, COMP interacts with growth factors and acts as a 'lattice' to present them for utilization by the cells (39). In the present study, expression levels of COMP decreased in co-cultured ASCs may be biological because the ASCs stopped proliferation and began differentiation when they were co-cultured with NPCs, while the cell number and activity may have been suppressed by differentiation compared with solely-cultured ASCs. However, the pathological decrease of COMP may hinder the ECM assembly and further weaken the cell attachment. Therefore, the role COMP plays in ASCs differentiation towards NP-like cell remains to be further elucidated.

LncRNAs have recently gained widespread attention as novel and crucial players in biological regulation. Wan et al investigated the differentially expressed lncRNAs in human intervertebral disc degeneration with 1ncRNA-mRNA microarray analysis (22). They found 1,052 lncRNAs and 1,314 mRNAs were differentially expressed between healthy human NP and degenerative NP tissues, of which the ENST00000461676 was the most significantly upregulated lncRNA with the fold-change at 136 and the NR_003716 was the top downregulated one with the fold-change at 
Table V. Top10 mRNAs, microRNAs and lncRNAs in ceRNA network.

\begin{tabular}{lllc}
\hline RNA symbol & Type & Style & Degree \\
\hline CDK6 & mRNA & Down & 7 \\
EHD3 & mRNA & Down & 6 \\
GSK3B & mRNA & Down & 6 \\
KPNA1 & mRNA & Down & 6 \\
ATP6V0E1 & mRNA & Down & 5 \\
FADS1 & mRNA & Down & 5 \\
ICMT & mRNA & Down & 5 \\
KRAS & mRNA & Down & 5 \\
NEO1 & mRNA & Down & 5 \\
NFASC & mRNA & Down & 5 \\
hsa-miR-27b-3p & miRNA & NO & 50 \\
hsa-miR-93-5p & miRNA & NO & 43 \\
hsa-miR-27a-3p & miRNA & NO & 30 \\
hsa-miR-98-5p & miRNA & NO & 30 \\
hsa-miR-140-3p & miRNA & NO & 18 \\
hsa-miR-21-3p & miRNA & NO & 15 \\
hsa-miR-202-3p & miRNA & NO & 14 \\
hsa-miR-146a-3p & miRNA & NO & 12 \\
hsa-miR-27b-5p & miRNA & NO & 12 \\
hsa-miR-455-3p & miRNA & NO & 12 \\
NR_002229 & lncRNA & Down & 19 \\
NR_003610 & lncRNA & Down & 16 \\
NR_024397 & lncRNA & Down & 11 \\
NR_015431 & lncRNA & Down & 8 \\
TCONS_00025860 & IncRNA & Down & 8 \\
TCONS_12_00014703 & lncRNA & Down & 8 \\
TCONS_00004015 & IncRNA & Down & 7 \\
TCONS_00017578 & lncRNA & Down & 7 \\
NR_001284 & lncRNA & Down & 6 \\
TCONS_12_00020760 & lncRNA & Down & 6 \\
\hline & & & \\
\hline
\end{tabular}

149 , despite the specific function of these IncRNAs in the IVDD not having been studied. Furthermore, we previously addressed the aberrantly expressed mRNAs and lncRNAs in degenerative NPCs co-cultured with ASCs, to determine the mRNAs and IncRNAs which may play a critical role during stem-cell-based therapy of IDD (12). In particular, we found that the secreted phosphoprotein 1 (SPP1), metallothionein $1 \mathrm{~F}$ (MT1F) and ectonucleotide pyrophosphatase 1 (ENPP1) presented the top three differentially expressed mRNAs by the degenerative NPCs, after they were co-cultured with ASCs.

Our data identified XIST (fold change $=23$ ) as the most significantly altered lncRNA in the co-culturing process. XIST, a 17-20 kb non-coding RNA, is essential for whole-chromosome silencing (43) via its inactivation of the X chromosome $(44,45)$. Additionally, XIST provides one of few tangible readouts of the stem cell quality and influences the pluripotent stem cell population (46). However, further investigations are required for a detailed functional characterization of XIST and other lncRNAs involved in NPC-directed differentiation of ASCs. Furthermore, the upregulation of IGF-1 and TGF $\beta$ was proven to protect the degeneration of IVD $(47,48)$; the IGF-1 and TGF $\beta-2$ was upregulated in the co-cultured ASCs in the present study (Fig. 4), indicating the protective function of ASCs to the NPCs. The expression of GPC3 was also upregulated in ASCs after co-culturing. GPC3 is known as a potential marker due to its higher gene expression and protein expression in NPCs compared with that in chondrocytes $(49,50)$. Therefore, the increment of GPC3 expression suggests ASCs differentiated towards NP-like cells.

Additionally, the TTTY15 was significantly downregulated in the current study (Fig. 4A), yet its role in intervertebral disc degeneration has not been investigated. TTTY15 had been demonstrated to regulate hypoxia-induced injury of vascular endothelial cells (51). Similarly, Huang et al found suppression of TTTY15 could mitigate hypoxia-induced injury in cardiomyocytes. Therefore, we speculate that the decrease of TTTY15 expression may indicate the tolerance of co-cultured ASCs to hypoxia, the condition that NPCs regularly undergo within intervertebral discs (52). The FABP3 expression was decreased after co-culturing (Fig. 4A). FABP3 binds to fatty acid for the intracellular lipid droplet accumulation, and it increases MSC survival $(53,54)$. It decreased possibly because the ASCs differentiated into NP-like cells after co-culturing. However, the contribution of these RNAs in the NPC-oriented differentiation of ASCs is currently unknown, and further investigation is required.

Regarding the ceRNA network, miR-27b-3p plays the most critical role in the network (degree of interaction $=50$ ), followed by hsa-miR-93-5p (degree of interaction $=43$ ). Specifically, miR-27b targets directly matrix metalloproteinase-13 (24), which is excessively expressed in degenerative disc tissue (55), and its downregulation leads to type II collagen loss and thus the development of IDD. Our data underline the critical role of miR-93 in the differentiation of ASCs into NP-like cells, which is in agreement with previous evidence. miR-93 was previously found to be expressed less in human degenerative NP tissue in a disc degeneration-dependent manner (25). These two miRNAs, miR-27b and miR-93, which are significantly altered during disc degeneration may also be markedly differentially expressed in NPC-guided differentiation of ASCs; however, further investigations are needed to validate any possible associations.

This study has several limitations. First, we investigated numerous RNAs via gene microarray assay but only validated results selectively by qPCR because of practicality and cost. In addition, we selectively interpreted the results based on previous studies and clinical implications. To the best of our knowledge, the present study was the second and the independent part of one project, which also included our previous study (12), which reported the differentially expressed mRNAs and lncRNAs between the NPCs cocultured with ASCs and the solely-cultured NPCs, thus the NPCs were not included as the control. Second, no further specific evaluations of particular roles were performed for NPC-directed differentiation of ASCs using overexpression or RNA interference. Similarly, the Path-net and the Signal-net were only outlined on the basis of a bioinformatics database, and comprehensive understanding of the specific key pathways or factors requires further sophisticated in vitro or in vivo studies. 
In conclusion, findings of the present study revealed differentially expressed mRNAs and lncRNAs in ASCs co-cultured with degenerative NPCs and highlights sophisticated cross-talking among mRNAs, lncRNAs, and miRNAs. Our data provide valuable information for the further development and clinical translation of stem cell-based approaches to treating IDD.

\section{Acknowledgements}

Not applicable.

\section{Funding}

This work was supported by the National Natural Science Foundation of China (no. 81301580).

\section{Availability of data and materials}

The datasets used and/or analyzed during the current study are available from the corresponding author on reasonable request.

\section{Authors' contributions}

ZH, QW and JWu conceptualized and designed the study. $\mathrm{ZH}, \mathrm{QW}, \mathrm{RG}, \mathrm{XW}$ and JWa performed the experiments and collected the data. ZH, LG, RG and JWu analyzed and interpreted the data. ZH, LG, XW and JWa drafted the manuscript. JWu supervised the study and revised the manuscript. All authors read and approved the final manuscript.

\section{Ethics approval and consent to participate}

All human tissues were obtained and used with the patients' informed consent and under the approval of Institutional Review Broad of the Shanghai General Hospital, Shanghai Jiao Tong University, Shanghai, China (approval no. 2019SQ098).

\section{Patient consent for publication}

Not applicable.

\section{Competing interests}

The authors declare that they have no competing interests.

\section{References}

1. Han Z, Gao L, Shi Q, Chen L and Chen C: Quantitative magnetic resonance imaging for diagnosis of intervertebral disc degeneration of the cervico-thoracic junction: A pilot study. Am J Transl Res 10: 925-935, 2018.

2. Chen P, Wu C, Huang M, Jin G, Shi Q, Han Z and Chen C: Apparent diffusion coefficient of diffusion-weighted imaging in evaluation of cervical intervertebral disc degeneration: An observational study with $3.0 \mathrm{~T}$ magnetic resonance imaging. Biomed Res Int 2018: 6843053, 2018.

3. Chen C, Jia Z, Han Z, Gu T, Li W, Li H, Tang Y, Wu J, Wang D, He Q and Ruan D: Quantitative T2 relaxation time and magnetic transfer ratio predict endplate biochemical content of intervertebral disc degeneration in a canine model. BMC Musculoskelet Disord 16: 157, 2015.

4. Urban JP and Roberts S: Degeneration of the intervertebral disc. Arthritis Res Ther 5: 120-130, 2003.
5. Andersson GB: Epidemiological features of chronic low-back pain. Lancet 354: 581-585, 1999.

6. Ruan D, Zhang Y, Wang D, Zhang C, Wu J, Wang C, Shi Z, $\mathrm{Xin} \mathrm{H}, \mathrm{Xu} \mathrm{C}, \mathrm{Li} \mathrm{H}$ and He Q: Differentiation of human Wharton's jelly cells toward nucleus pulposus-like cells after coculture with nucleus pulposus cells in vitro. Tissue Eng Part A 18: 167-175, 2012.

7. Zeng Y, Chen C, Liu W, Fu Q, Han Z, Li Y, Feng S, Li X, Qi C, $\mathrm{Wu} \mathrm{J}$, et al: Injectable microcryogels reinforced alginate encapsulation of mesenchymal stromal cells for leak-proof delivery and alleviation of canine disc degeneration. Biomaterials 59: 53-65, 2015.

8. Han Z, Zhang Y, Gao L, Jiang S and Ruan D: Human Wharton's jelly cells activate degenerative nucleus pulposus cells in vitro. Tissue Eng Part A 24: 1035-1043, 2018.

9. Ying J, Han Z, Zeng Y, Du Y, Pei S, Su L, Ruan D and Chen C: Evaluation of intervertebral disc regeneration with injection of mesenchymal stem cells encapsulated in PEGDA-microcryogel delivery system using quantitative T2 mapping: A study in canines. Am J Transl Res 11: 2028-2041, 2019.

10. Ying J, Han Z, Pei S, Su L and Ruan D: Effects of stromal cell-derived factor- $1 \alpha$ secreted in degenerative intervertebral disc on activation and recruitment of nucleus pulposus-derived stem cells. Stem Cells Int 2019: 9147835, 2019.

11. Zhang Y, Tao H, Gu T, Zhou M, Jia Z, Jiang G, Chen C, Han Z, $\mathrm{Xu} \mathrm{C}$, Wang D, et al: The effects of human Wharton's jelly cell transplantation on the intervertebral disc in a canine disc degeneration model. Stem Cell Res Ther 6: 154, 2015.

12. Han Z, Wang J, Gao L, Wang Q and Wu J: Aberrantly expressed messenger RNAs and long noncoding RNAs in degenerative nucleus pulposus cells co-cultured with adipose-derived mesenchymal stem cells. Arthritis Res Ther 20: 182, 2018.

13. Sun Z, Liu ZH, Zhao XH, Sun L, Chen YF, Zhang WL, Gao Y, Zhang YZ, Wan ZY, Samartzis D, et al: Impact of direct cell co-cultures on human adipose-derived stromal cells and nucleus pulposus cells. J Orthop Res 31: 1804-1813, 2013.

14. Sakai D, Mochida J, Iwashina T, Watanabe T, Nakai T, Ando K and Hotta T: Differentiation of mesenchymal stem cells transplanted to a rabbit degenerative disc model: Potential and limitations for stem cell therapy in disc regeneration. Spine (Phila Pa 1976) 30: 2379-2387, 2005.

15. Chun HJ, Kim YS, Kim BK, Kim EH, Kim JH, Do BR, Hwang SJ, Hwang JY and Lee YK: Transplantation of human adipose-derived stem cells in a rabbit model of traumatic degeneration of lumbar discs. World Neurosurg 78: 364-371, 2012.

16. Sivakamasundari V and Lufkin T: Stemming the degeneration: IVD stem cells and stem cell regenerative therapy for degenerative disc disease. Adv Stem Cells 2013: 724547, 2013.

17. Hung $\mathrm{T}$ and Chang HY: Long noncoding RNA in genome regulation: Prospects and mechanisms. RNA Biol 7: 582-585, 2010.

18. Mercer TR, Dinger ME and Mattick JS: Long non-coding RNAs: Insights into functions. Nat Rev Genet 10: 155-159, 2009.

19. Yang L, Froberg JE and Lee JT: Long noncoding RNAs: Fresh perspectives into the RNA world. Trends Biochem Sci 39: 35-43, 2014.

20. Batista PJ and Chang HY: Long noncoding RNAs: Cellular address codes in development and disease. Cell 152: 1298-1307, 2013

21. Liu X, Che L, Xie YK, Hu QJ, Ma CJ, Pei YJ, Wu ZG, Liu ZH, Fan LY and Wang HQ: Noncoding RNAs in human intervertebral disc degeneration: An integrated microarray study. Genom Data 5: 80-81, 2015.

22. Wan ZY, Song F, Sun Z, Chen YF, Zhang WL, Samartzis D, Ma CJ, Che L, Liu X, Ali MA, et al: Aberrantly expressed long noncoding RNAs in human intervertebral disc degeneration: A microarray related study. Arthritis Res Ther 16: 465, 2014.

23. Ambros V: The functions of animal microRNAs. Nature 431: 350-355, 2004.

24. Li HR, Cui Q, Dong ZY, Zhang JH, Li HQ and Zhao L: Downregulation of MIR-27b is involved in loss of Type II collagen by directly targeting matrix metalloproteinase 13 (MMP13) in human intervertebral disc degeneration. Spine (Phila Pa 1976) 41: E116-E123, 2016.

25. Jing W and Jiang W: MicroRNA-93 regulates collagen loss by targeting MMP3 in human nucleus pulposus cells. Cell Prolif 48: 284-292, 2015.

26. Li Z, Yu X, Shen J, Chan MT and Wu WK: MicroRNA in intervertebral disc degeneration. Cell Prolif 48: 278-283, 2015.

27. Yan N, Yu S, Zhang $\mathrm{H}$ and Hou T: Lumbar disc degeneration is facilitated by MiR-100-mediated FGFR3 suppression. Cell Physiol Biochem 36: 2229-2236, 2015. 
28. Russo V, Yu C, Belliveau P, Hamilton A and Flynn LE: Comparison of human adipose-derived stem cells isolated from subcutaneous, omental, and intrathoracic adipose tissue depots for regenerative applications. Stem Cells Transl Med 3: 206-217, 2014.

29. Schaffler A and Buchler C: Concise review: Adipose tissue-derived stromal cells-basic and clinical implications for novel cell-based therapies. Stem Cells 25: 818-827, 2007.

30. Eischen-Loges M, Oliveira KMC, Bhavsar MB, Barker JH and Leppik L: Pretreating mesenchymal stem cells with electrical stimulation causes sustained long-lasting pro-osteogenic effects. PeerJ 6: e4959, 2018.

31. Chen J, Xu J, Li Y, Zhang J, Chen H, Lu J, Wang Z, Zhao X, Xu K, Li Y, et al: Competing endogenous RNA network analysis identifies critical genes among the different breast cancer subtypes. Oncotarget 8: 10171-10184, 2017.

32. Jonas $S$ and Izaurralde E: Towards a molecular understanding of microRNA-mediated gene silencing. Nat Rev Genet 16: 421-433, 2015.

33. Li CY, Liang GY, Yao WZ, Sui J, Shen X, Zhang YQ, Peng H, Hong WW, Ye YC, Zhang ZY, et al: Integrated analysis of long non-coding RNA competing interactions reveals the potential role in progression of human gastric cancer. Int J Oncol 48 : 1965-1976, 2016

34. Zhang W and Liu HT: MAPK signal pathways in the regulation of cell proliferation in mammalian cells. Cell Res 12: 9-18, 2002.

35. Zhang K, Ding W, Sun W, Sun XJ, Xie YZ, Zhao CQ and Zhao J: Betal integrin inhibits apoptosis induced by cyclic stretch in annulus fibrosus cells via ERK1/2 MAPK pathway. Apoptosis 21: $13-24,2016$.

36. Niu CC, Lin SS, Yuan LJ, Chen LH, Wang IC, Tsai TT, Lai PL and Chen WJ: Hyperbaric oxygen treatment suppresses MAPK signaling and mitochondrial apoptotic pathway in degenerated human intervertebral disc cells. J Orthop Res 31: 204-209, 2013.

37. Tian Y, Yuan W, Fujita N, Wang J, Wang H, Shapiro IM and Risbud MV: Inflammatory cytokines associated with degenerative disc disease control aggrecanase-1 (ADAMTS-4) expression in nucleus pulposus cells through MAPK and NF-кB. Am J Pathol 182: 2310-2321, 2013.

38. Li P, Xu Y, Gan Y, Wang L, Ouyang B, Zhang C, Luo L, Zhao C and Zhou Q: Estrogen enhances matrix synthesis in nucleus pulposus cell through the estrogen receptor $\beta$-p38 MAPK pathway. Cell Physiol Biochem 39: 2216-2226, 2016.

39. Acharya C, Yik JH, Kishore A, Van Dinh V, Di Cesare PE and Haudenschild DR: Cartilage oligomeric matrix protein and its binding partners in the cartilage extracellular matrix: Interaction, regulation and role in chondrogenesis. Matrix Biol 37: 102-111, 2014.

40. Rosenberg K, Olsson H, Morgelin M and Heinegard D: Cartilage oligomeric matrix protein shows high affinity zinc-dependent interaction with triple helical collagen. J Biol Chem 273: 20397-20403, 1998.

41. Chen FH, Herndon ME, Patel N, Hecht JT, Tuan RS and Lawler J: Interaction of cartilage oligomeric matrix protein/ thrombospondin 5 with aggrecan. J Biol Chem 282: 24591-24598, 2007.

42. Chen FH, Thomas AO, Hecht JT, Goldring MB and Lawler J: Cartilage oligomeric matrix protein/thrombospondin 5 supports chondrocyte attachment through interaction with integrins. J Biol Chem 280: 32655-32661, 2005 .
43. Penny GD, Kay GF, Sheardown SA, Rastan S and Brockdorff N: Requirement for Xist in X chromosome inactivation. Nature 379: 131-137, 1996.

44. Brockdorff N, Ashworth A, Kay GF, McCabe VM, Norris DP, Cooper PJ, Swift S and Rastan S: The product of the mouse Xist gene is a $15 \mathrm{~kb}$ inactive $\mathrm{X}$-specific transcript containing no conserved ORF and located in the nucleus. Cell 71: 515-526, 1992.

45. Clemson CM, McNeil JA, Willard HF and Lawrence JB: XIST RNA paints the inactive $X$ chromosome at interphase: Evidence for a novel RNA involved in nuclear/chromosome structure. J Cell Biol 132: 259-275, 1996.

46. Minkovsky A, Patel S and Plath K: Concise review: Pluripotency and the transcriptional inactivation of the female mammalian $X$ chromosome. Stem Cells 30: 48-54, 2012.

47. Kennon JC, Awad ME, Chutkan N, DeVine J and Fulzele S: Current insights on use of growth factors as therapy for intervertebral disc degeneration. Biomol Concepts 9: 43-52, 2018.

48. Gruber HE, Hoelscher GL, Ingram JA, Bethea S and Hanley EN: IGF-1 rescues human intervertebral annulus cells from in vitro stress-induced premature senescence. Growth Factors 26: 220-225, 2008.

49. Yuan M, Yeung CW, Li YY, Diao H, Cheung KMC, Chan D, Cheah K and Chan PB: Effects of nucleus pulposus cell-derived acellular matrix on the differentiation of mesenchymal stem cells. Biomaterials 34: 3948-3961, 2013.

50. Minogue BM, Richardson SM, Zeef LA, Freemont AJ and Hoyland JA: Characterization of the human nucleus pulposus cell phenotype and evaluation of novel marker gene expression to define adult stem cell differentiation. Arthritis Rheum 62 : 3695-3705, 2010

51. Zheng J, Zhuo YY, Zhang C, Tang GY, Gu XY and Wang F: LncRNA TTTY15 regulates hypoxia-induced vascular endothelial cell injury via targeting miR-186-5p in cardiovascular disease. Eur Rev Med Pharmacol Sci 24: 3293-3301, 2020.

52. Huang S, Tao W, Guo Z, Cao J and Huang X: Suppression of long noncoding RNA TTTY15 attenuates hypoxia-induced cardiomyocytes injury by targeting miR-455-5p. Gene 701: 1-8, 2019.

53. Wang S, Zhou Y, Andreyev O, Hoyt RF Jr, Singh A, Hunt T and Horvath KA: Overexpression of FABP3 inhibits human bone marrow derived mesenchymal stem cell proliferation but enhances their survival in hypoxia. Exp Cell Res 323: 56-65, 2014.

54. Bensaad K, Favaro E, Lewis CA, Peck B, Lord S, Collins JM, Pinnick KE, Wigfield S, Buffa FM, Li JL, et al: Fatty acid uptake and lipid storage induced by HIF-1 $\alpha$ contribute to cell growth and survival after hypoxia-reoxygenation. Cell Rep 9: 349-365, 2014.

55. Weiler C, Nerlich AG, Zipperer J, Bachmeier BE and Boos N: 2002 SSE Award Competition in Basic Science: Expression of major matrix metalloproteinases is associated with intervertebral disc degradation and resorption. Eur Spine J 11: 308-320, 2002.

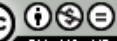

This work is licensed under a Creative Commons Attribution-NonCommercial-NoDerivatives 4.0 International (CC BY-NC-ND 4.0) License. 